ENSV TEADUSTE AKADEEMIA TOIMETISED. XVI KOIDE

KEEMIA * GEOLOOGIA. 1967, Nr. 2

ИЗВЕСТИЯ АКАДЕМИИ НАУК ЭСТОНСКОИ ССР. ТОМ ХVI

ХИМИЯ * ГЕОЛОГИЯ. 1967, ㅅ․ 2

\title{
ПРИМЕНЕНИЕ ГАРМОНИЧЕСКОГО АНАЛИЗА ДЛЯ ИЗУЧЕНИЯ ДИНАМИКИ НАПОРА И СТОКА ОДНОМЕРНОГО ПОТОКА ПОДЗЕМНЫХ ВОД С НАПОРНОИ ПОВЕРХНОСТЬЮ
}

В статье рассматриваются возможности применения гармонического анализа для изучения распределения гидравлического напора, градиента напора и естественных ресурсов одномерного потока подземных вод с напорной поверхностью, подвергавшегося нестационарному режиму фильтрации. При этом учитываются длина, параметры ироницаемости, уклон пьезометрической поверхности и условия питания и разгрузки ведоносного горизонта.

\section{Постановка проблемы}

Распределение гидравлического напора в водоносном горизонте, вмещающем подземные воды с напорной поверхностью, выражается, согласно теории $\left[{ }^{1-4}\right]$, дифференциальным уравнением

$$
\begin{gathered}
\partial\left(K_{1} \partial p / \partial x\right) / \partial x+\partial\left(K_{2} \partial p / \partial y\right) / \partial y+\partial\left(K_{3} \partial p / \partial z\right) / \partial z+ \\
+A(x, y, z, t)=\beta^{*} \partial p / \partial t
\end{gathered}
$$

где $p$ - гидравлический напор; $K_{1}, K_{2}, K_{3}-$ динамические коэффициенты фильтрации и $\beta^{*}-$ коэффициент упругоемкости пласта, причем $K_{1}, K_{2}, K_{3}$ и $\beta^{*}$ могут зависеть от $x, y, z$.

Функция $A(x, y, z, t)$ описывает некоторый внутренний положительный или отрицательный источник системы. Примером такого источника может служить действие родника, отбор или нагнетание воды при помощи буровой скважины, перетекание через слабопроницаемые раздельные слои, освобождение поровых вод глинистых прослоек вследствие дегидратации при сильном понижении напора в горизонте [5] и т. п.

Рассмотрим водоносный горизонт, содержащий подземные воды с напорной поверхностью, внутренний и внешний источник в котором отсутствует, а водоупорные слои можно счнтать абсолютно непроницаемыми. Если при этом распределение гидравлического напора зависит только от одной пространственной координаты, т. е. мощность и гидравлические параметры пласта можно считать с достаточно хорошим приближением постоянными, то уравнение (1) упрощается и принимает вид

$$
\partial p / \partial t=x \partial^{2} p / \partial x^{2},
$$

где $x>0-$ коэффициент пьезопроводности.

Дифференциальное уравнение (2) описывает напорную одномерную - фильтрацию. 
Ниже изучается нестационарный режим фильтрации потока подземных вод с напорной поверхностью для нахождения функции распределения гидравлического напора $p(x, t)$ в виде решения уравнения (2) при граничных условиях

$$
p_{x=0}=p_{D}(t),\left.\quad p\right|_{x=l}=p_{T}(t)
$$

и начальном условии

$$
\left.p\right|_{t=0}=p_{D}(0)+\left[p_{T}(0)-p_{D}(0)\right] x / l,
$$

где $p_{D}$ - напор в области питания и $p_{T}$ - в области разгрузки потока; $l$ - расстояние между областями пнтания и разгрузки.

Здесь принято, что поток подземных вод в сечении $x=l$ пополняется за счет инфильтрации вод атмосферных осадков и т. п., а в сечении $x=l$ разгружается в какой-то поверхностный водоем или в другой водоносный горизонт (рис. $1, a-8$ ). Поэтому $p_{D}$ и $p_{T}$ являются некоторыми функциями времени гидрогеолого-метеорологических факторов и выражаются изменениями гидравлического напора $p_{D}(t)$ и $p_{T}(t)$ соответственно в области питания и разгрузки изучаемого потока.

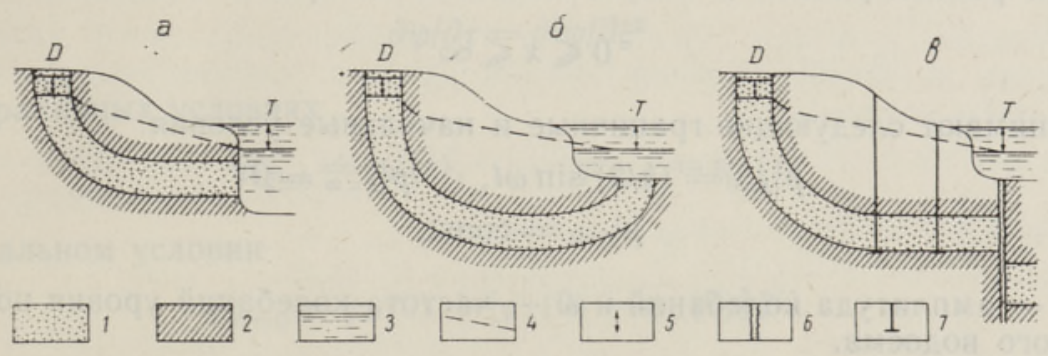

Рис. 1. Схемы питания и разгрузки потока подземных вод. Виды разгрузки: $a$ - у берегов поверхностного водоема; $\sigma$ - за пределами берегов поверхностного водоема; $в$ - через тектоническую трещину. $I$ - водоносный горнзонт; 2 - водоупорный слой; 3 - поверхностный водоем: 4 - пьезометрическая поверхность при стационарном режиме фильтрации (изображена по отношению к некоторой горизонтальной плоскости сравнения): 5 амплитуда колебання уровня воды в области питания $(D)$ и разгрузки $(T)$ : 6 - тектоническая трешина; 7 - буровая наблюдательная скважина.

Начальное условие (4) принято на основе предположения, что при $t \leqslant 0$ подземный поток подчиняется стационарному режиму фильтрации $\left(p_{D}(t), p_{T}(t)=\mathrm{const}\right)$ и в соответствии с известным законом Дарси представляет собой линейную функцию от $x$.

Для изучения динамики разгрузки потока подземных вод придется найти градиент напора в месте разгрузки, то есть величину $\partial p(x, t) /\left.\partial x\right|_{x=l}$. Это особенно важно в случае, когда поток подземных ьод разгружается в поверхностный водоем.

Колебание уровня поверхностных вод происходит во времени более интенсивно, чем перераспределение гидравлического напора в водовмещающем пласте. Поэтому функция $\partial p(x, t) / \partial x$ вблизи края $x=0$ чувствительна к изменениям граничного условия $p_{T}(t)$. Иногда колебания $p_{T}(t)$ при определенных значениях коэффициента пьезопроводности и пьезометрического уклона могут вызывать даже перемену знака функции $\partial p(x, t) / \partial x$.

Следовательно, режим фильтрации в водоносном горизонте существенно зависит от переменного подпора, созданного колебанием уровня 
поверхностных вод в области разгрузки. Так как это явление имеет в гидрогеологии большое значение, назовем его, в порядке предложения, подпорным регулированием стока подземных вод.*

Если под естественными ресурсами подземных вод понимать расход водоносного горизонта на всей площади его распространения [6], то, на основе закона Дарси, естественные ресурсы горизонта можно представить интегралом

$$
Q=-F K \int_{t_{1}}^{t_{2}} \partial p(x, t) /\left.\partial x\right|_{x=l} d t
$$

где $Q$ - естественные ресурсы подземных вод за некоторый промежуток времени от $t_{1}$ до $t_{2} ; F-$ площадь поперечного сечения потока и $K-$ коэффициент фильтрации.

Судя по доступной литературе $\left[{ }^{2}\right]$, до сих пор в гидрогеологии существуют только некоторые частичные решения выдвинутой выше проблемы.

Во избежание математических трудностей поток подземных вод обычнс рассматривают в полубесконечном интервале изменений

$$
0 \leqslant x \leqslant \infty
$$

и принимают следующие граничные и начальные условия:

$$
\begin{gathered}
\left.p\right|_{x=0}=(h / 2) \sin \omega t,\left.\quad p\right|_{x=\infty}=0 \\
\left.p\right|_{t=0}=\text { const, }
\end{gathered}
$$

где $h$ - амплитуда колебаний и $\omega-$ частота колебаний уровня поверхностного водоема.

При таком способе постановки задачи теряется возможность учитывать условия питания, наклон пьезометрической поверхности и расстояние между областями питания и разгрузки, под влиянием которых формируются многие важные особенности гидродинамического режима потока подземных вод. Очевидно, аппроксимация колебания уровня поверхностного водоема синусоидой (7) весьма далека от естественного.

Поэтому решения уравнения (2) при допущениях (7), (8) неудовлетворительно отражают природную обстановку и пригодны для изучения сравнительно узкого круга теоретических вопросов.

\section{Решение проблемы}

Решения поставленной задачи целесообразно выразить, в интересах их наиболее универсального использования, через безразмерные параметры. Вместо вышеприведенных величин, имеющих размерности

* В литературе, посвященной исследованиям динамики потоков грунтовых вод, имеющих свободную поверхность, широко применяется понятие «береговое регулирование поверхностного стока», под которым подразумевается инфильтрация речных вод в берега во время восходящей стадии половодья и возврат их в реку при спаде последнего [6]. Приведенное в тексте определение имеет, по нашему мнению, более широкое гидрогеологическое содержание. В частности оно применимо и в отношении потоков подземных вод с напорной поверхностью, которые могут разгружаться далеко в море, в большие озера или через тектонические трещины непосредственно в ложе реки (см. рис. $1,6,8)$, т. е. по существу за пределами берегов поверхностнюго водоема. 


$$
\begin{gathered}
{[x t]=\left[L^{2}\right]} \\
{[l]=[L]} \\
{[p]=[L]}
\end{gathered}
$$

$$
\begin{array}{r}
{\left[p_{D}\right]=[L]} \\
{\left[p_{T}\right]=[L]} \\
{[x]=[L],}
\end{array}
$$

введем безразмерные:

$$
\begin{aligned}
x t / \Lambda^{2} & =\tau \\
l / \Lambda & =\lambda \\
p / \Lambda & =\psi
\end{aligned}
$$

$$
\begin{gathered}
p_{D} / \Lambda=\psi_{D} \\
p_{T} / \Lambda=\psi_{T} \\
x / \Lambda=\xi,
\end{gathered}
$$

где $\tau, \lambda, \xi-$ соответственно безразмерное время, расстояние, текучее расстояние и $\psi, \psi_{D}, \psi_{T}$ - безразмерные напоры.

Отметим, что переход от безразмерных величин к размерным легко осуществляется, если $[\Lambda]=[L]$ выбирать так, чтобы она численно равнялась единице.

В безразмерных величинах поставленная задача может быть сформулирована следующим образом:

найти решение дифференциального уравнения

$$
\partial \psi / \partial \tau=\partial^{2} \psi / \partial \xi^{2}
$$

при граничных условиях

$$
\left.\psi\right|_{\xi=0}=\psi_{D}(\tau),\left.\quad \psi\right|_{\xi=\lambda}=\psi_{T}(\tau)
$$

и начальном условии

$$
\left.\psi\right|_{\tau=0}=\psi_{D}(0)+\left[\psi_{T}(0)-\psi_{D}(0)\right] \xi / \lambda .
$$

При краевых условиях (22) и (23) можно в промежутке

$$
0 \leqslant \xi \leqslant \lambda
$$

найти приближенное решение уравнения (21) при помощи известных численных методов (метод сеток, метод прямых и т. д.) или в виде отрезка некоторого тригонометрического ряда. Ниже используется метод гармонического анализа, поскольку полученное таким образом аналитическое решение нагляднее и имеет преимущество с точки зрения последующих теоретических исследований проблем режима подземных вод.

Найдем частные решения уравнения (21). методом Фурье. Ищем их в форме

$$
\psi(\xi, \tau)=w(\xi, \tau)+u(\xi, \tau),
$$

где $w(\xi, \tau)$ - некоторая линейная по $\xi$ функция, удовлетворяющая граничным условиям (22) и начальному условию $(23)$, а $u(\xi, \tau)$ функция, удовлетворяющая нулевым граничным и начальным условиям.

Функцию $w(\xi, \tau)$ выберем в форме

$$
w(\xi, \tau)=\psi_{D}(\tau)+\left[\psi_{T}(\tau)-\psi_{D}(\tau)\right] \xi / \lambda .
$$

Подставляя (25) в уравнение (21) и учитывая (26), получим для $u(\xi, \tau)$ неоднородное уравнение, решение которого ищем в виде ряда 


$$
u(\xi, \tau)=\sum_{i=1}^{\infty} v_{i}(\tau) \sin i \pi \xi / \lambda,
$$

где функции $v_{i}(\tau)$ должны удовлетворять условию $\tau_{i}(0)=0 \quad(i=1$, $2,3, \ldots, \infty)$ и уравнению

$$
\begin{aligned}
& \sum_{i=1}^{\infty}\left[\partial v_{i}(\tau) / \partial \tau+(i \pi / \lambda)^{2} v_{i}(\tau)\right] \sin i \pi \xi / \lambda= \\
& =-\partial\left\{\psi_{D}(\tau)+\left[\psi_{T}(\tau)-\psi_{D}(\tau)\right] \xi / \lambda\right\} / \partial \tau .
\end{aligned}
$$

Умножая обе стороны уравнения (28) на $\sin j \pi \xi / \lambda$ и интегрируя по $\xi$ в промежутке от 0 до $\lambda$, получим в силу ортогональности тригонометрической системы при $j=i$

$$
\partial v_{i}(\tau) / \partial \tau+(i \pi / \lambda)^{2} v_{i}(\tau)=-r_{D i} \partial \psi_{D}(\tau) / \partial \tau-r_{T i} \partial \psi_{T}(\tau) / \partial \tau
$$

где

$$
r_{D i}=2 / i \pi
$$

H

$$
r_{T i}=(-1)^{i+1} 2 / i \pi
$$

Решение линейного неоднородного дифференциального уравнения построим на основе решения соответствующего однородного дифференциального уравнения

$$
v_{i}=C \exp \left[-(i \pi / \lambda)^{2} \tau\right]
$$

методом вариации постоянных в форме

$$
v_{i}=G_{i}(\tau) \exp \left[-(i \pi / \lambda)^{2} \tau\right] .
$$

Тогда

$$
\partial v_{i} / \partial \tau=\left[\partial G_{i}(\tau) / \partial \tau\right] \exp \left[-(i \pi / \lambda)^{2} \tau\right]-G_{i}(\tau)(i \pi / \lambda)^{2} \exp \left[-(i \pi / \lambda)^{2} \tau\right] .
$$

Подставляя (33) и (34) в уравнение (29), имеем

$$
\begin{gathered}
\partial G_{i}(\tau) / \partial \tau=-\left[r_{D i} \partial \psi_{D}(\tau) / \partial \tau\right] \exp \left[(i \pi / \lambda)^{2} \tau\right]- \\
-\left[r_{T i} \partial \psi_{T}(\tau) / \partial \tau\right] \exp \left[(i \pi / \lambda)^{2} \tau\right] .
\end{gathered}
$$

Интегрированием по частям получим из уравнения (35):

$$
\begin{gathered}
G_{i}(\tau)=-r_{D i}\left\{\psi_{D}(\tau) \exp \left[(i \pi / \lambda)^{2} \tau\right]-\psi_{D}(0)-\right. \\
\left.-(i \pi / \lambda)^{2} \int_{0}^{\tau} \psi_{D}(\Theta) \exp \left[(i \pi / \lambda)^{2} \Theta\right] d \Theta\right\}-r_{T i}\left\{\psi_{T}(\tau) \exp \left[(i \pi / \lambda)^{2} \tau\right]-\right. \\
\left.-\psi_{T}(0)-(i \pi / \lambda)^{2} \int_{0}^{\tau} \psi_{T}(\Theta) \exp \left[(i \pi / \lambda)^{2} \Theta\right] d \Theta\right\} .
\end{gathered}
$$

Легко убедиться в том, что при $\tau=0$ функции $G_{i}(0)=0$ и, следовательно, $v_{i}$ удовлетворяют условию $v_{i}(0)=0$.

На основе $(25),(26),(30),(31),(33)$ и (36) получим искомое решение в виде

$$
\begin{gathered}
\psi(\xi, \tau)=\psi_{D}(\tau)+\left[\psi_{T}(\tau)-\psi_{D}(\tau)\right] \xi / \lambda+\sum_{i=1}^{\infty}(2 / i \pi) \sin i \pi \xi / \lambda \times \\
\times\left\{( - 1 ) ^ { i } \left[\psi_{T}(\tau)-\psi_{T}(0) \exp \left[-(i \pi / \lambda)^{2} \tau\right]-\right.\right.
\end{gathered}
$$




$$
\left.-(i \pi / \lambda)^{2} \int_{0}^{\tau} \psi_{T}(\Theta) \exp \left[(i \pi / \lambda)^{2}(\Theta-\tau)\right] d \Theta\right]-\left[\psi_{D}(\tau)-\right.
$$

$$
\left.\left.-\psi_{D}(0) \exp \left[-(i \pi / \lambda)^{2} \tau\right]-(i \pi / \lambda)^{2} \int_{0}^{\tau} \psi_{D}(\Theta) \exp \left[(i \pi / \lambda)^{2}(\Theta-\tau)\right] d \theta\right]\right\}
$$

Ряд в выражении (37), вообще говоря, не сходится равномерно, и его дифференцирование по $\xi$ может оказаться невозможным. Отсюда вытекает, что неформальное решение поставленной задачи -- найти $\partial \psi(\xi, \tau) /\left.\partial \xi\right|_{\xi=\text {. }}$ методами гармонического анализа - существует только при некоторых определенных свойствах граничных условий $\psi_{D}(\tau)$ и $\psi_{T}(\tau)$.

Выясним эти свойства.

Интегрируя выражение (35) снова по частям; получим *

$$
\begin{gathered}
G_{i}(\tau)=(\lambda / i \pi)^{2}\left\{-r_{D i}\left[\left[\partial \psi_{D}(\tau) / \partial \tau\right] \exp \left[(i \pi / \lambda)^{2} \tau\right]-\partial \psi_{D}(0) / \partial \tau-\right.\right. \\
\left.-\int_{0}^{\tau}\left[\partial^{2} \psi_{D}(\Theta) / \partial \Theta^{2}\right] \exp \left[(i \pi / \lambda)^{2} \Theta\right] d \Theta\right]-r_{T i}\left[\left[\partial \psi_{T}(\tau) / \partial \tau\right] \exp \left[(i \pi / \lambda)^{2} \tau\right]-\right. \\
\left.\left.-\partial \psi_{T}(0) / \partial \tau-\int_{0}^{\tau}\left[\partial^{2} \psi_{T}(\Theta) / \partial \Theta^{2}\right] \exp \left[(i \pi / \lambda)^{2} \Theta\right] d \theta\right]\right\}
\end{gathered}
$$

После подстановки (30), (31) и (38) в формулу (25) получим решение в новом виде:

$$
\begin{gathered}
\psi(\xi, \tau)=\psi_{D}(\tau)+\left[\psi_{T}(\tau)-\psi_{D}(\tau)\right] \xi / \lambda+\sum_{i=1}^{\infty}\left[\left(2 \lambda^{2} \sin i \pi \xi / \lambda\right) /(i \pi)^{3}\right] \times \\
\times\left\{\partial\left[(-1)^{i} \psi_{T}(\tau)-\psi_{D}(\tau)\right] / \partial \tau-\left[\partial\left[(-1)^{i} \psi_{T}(0)-\psi_{D}(0)\right] / \partial \tau\right] \times\right. \\
\times \exp \left[-(i \pi / \lambda)^{2} \tau\right]-\int_{0}^{\tau}\left[\partial ^ { 2 } \left[(-1)^{i} \psi_{T}(\Theta)-\right.\right. \\
\left.\left.\left.-\psi_{D}(\Theta)\right] / \partial \Theta^{2}\right] \exp \left[(i \pi / \lambda)^{2}(\Theta-\tau)\right] d \Theta\right\}
\end{gathered}
$$

в котором ряд сходится равномерно. Значит, условием равномерной сходимости рассматриваемых рядов является дважды непрерывная дифференцируемость граничных условий $\psi_{D}(\tau)$ и $\psi_{T}(\tau)$.

По известным теоремам анализа можно полученные ряды почленно дифференцировать по $\xi$ и при $\xi=\lambda$ будем иметь выражение для грядиента напора на месте разгрузки потока:

$$
\begin{gathered}
\partial \psi(\xi, \tau) /\left.\partial \xi\right|_{\xi=\lambda}=\left[\psi_{T}(\tau)-\psi_{D}(\tau)\right] / \lambda+\sum_{i=1}^{\infty}\left[2 \lambda /(i \pi)^{2}\right] \times \\
\times\left\{\partial\left[\psi_{T}(\tau)-(-1)^{i} \psi_{D}(\tau)\right] / \partial \tau-\right. \\
-\left[\partial\left[\psi_{T}(0)-(-1)^{i} \psi_{D}(0)\right] / \partial \tau\right] \exp \left[-(i \pi / \lambda)^{2} \tau\right]- \\
\left.-\int^{\tau}\left[\partial^{2}\left[\psi_{T}(\Theta)-(-1)^{i} \psi_{D}(\Theta)\right] / \partial \Theta^{2}\right] \exp \left[(i \pi / \lambda)^{2}(\Theta-\tau)\right] d \Theta\right\} .
\end{gathered}
$$

Безразмерные естественные ресурсы подземных вод за промежуток времени от 0 до $\tau$ в горизонте постоянной мощности и проницаемости можно представить на основе выражения (5) в виде

* Для простоты обозначим: $\partial \psi_{D}(\tau) /\left.\partial \tau\right|_{\tau=0}=\partial \psi_{D}(0) / \partial \tau$ и $\partial \psi_{T}(\tau) /\left.\partial \tau\right|_{\tau=0}=\partial \psi_{T}(0) / \partial \tau$. 


$$
\begin{gathered}
\Omega=\Phi K \int_{0}^{\tau}\left\{\left[\psi_{D}(\theta)-\psi_{T}(\theta)\right] / \lambda-\sum_{i=1}^{\infty}\left[2 \lambda /(i \pi)^{2}\right] \times\right. \\
X\left\{\partial\left[\psi_{T}(\theta)-(-1)^{i} \psi_{D}(\Theta)\right] / \partial \theta-\right. \\
-\left[\partial\left[\psi_{T}(0)-(-1)^{i} \psi_{D}(0)\right] / \partial \theta\right] \exp \left[-(i \pi / \lambda)^{2} \theta\right]- \\
\left.-\int_{0}^{\tau}\left[\partial^{2}\left[\psi_{T}(\vartheta)-(-1)^{i} \psi_{D}(\vartheta)\right] / \partial \vartheta^{2}\right] \exp \left[(i \pi / \lambda)^{2}(\vartheta-\theta)\right] d \vartheta\right\} d \theta
\end{gathered}
$$

где $\Phi K-$ безразмерное произведение $F K$.

Таким образом, выражения (39), (40) и (41) представляют аналитическое решение поставленной проблемы.

\section{Методы вычисления и замечания}

Вышеизложенные решения (39), (40) и (41) содержат функциональные ряды, часть которых находится под знаком интеграла. Их вычисление вручную с необходимой точностью весьма трудоемкая работа гораздо целесообразнее пользоваться для этого быстродействующей вычислительной машиной.

Применение возможностей машинного вычисления позволяет, кроме того, включить в основную программу вычислений подпрограммы, которые надлежащим образом обрабатывают исходные данные, сглаживают в них влияние случайностей и неточностей наблюдений.

Здесь надо иметь в виду следующее обстоятельство.

Хотя природные граничные условия в сущности являются непрерывными и достаточно гладкими функциями времени и имеют в каждой точке производную, они заданы некоторой совокупностью дискретных значений наблюдений водомерных постов, т. е. таблично. Это затрудняет решение с моделированием природных граничных условий, так как использование выражений (39), (40), и (41) требует вычисления произьодных $\partial / \partial \tau$ и $\partial^{2} / \partial \tau^{2}$ от функций $\psi_{D}(\tau)$ и $\psi_{T}(\tau)$.

Для преодоления трудностей, связанных с дифференцированием и последующим интегрированием эмпирических функций $\psi_{D}(\tau)$ и $\psi_{T}(\tau)$, целесообразно их аппроксимировать полиномами Чебышева. Такой прием может оказаться особенно полезным в том случае, если решение требуется на основе многолетних рядов наблюдений. Тогда, с точки зрения гидрогеологической практики, основой вычислений должен служить не ряд наблюдений некоторого конкретного года, а обобщенная кривая с определенной обеспеченностью *.

По-видимому, можно составить соответствующую подпрограмму, при помощи которой определяются коэффициенты полинома так, чтобы последний аппроксимировал совокупность наблюдений в заданном интервале обеспеченности.

Аппроксимируем граничные условия $\psi_{D}(\tau)$ и $\psi_{T}(\tau)$ полиномами шестой степени:

$$
\psi_{D}(\tau)=b_{D 0}+b_{D 1} \tau+b_{D 2} \tau^{2}+b_{D 3} \tau^{3}+b_{D 4} \tau^{4}+b_{D 5} \tau^{5}+b_{D 6} \tau^{6}
$$

* Обеспеченность гидрологической величины (напр., уровня воды) - вероятность повышения рассматриваемого значения гидрологической величины среди совокупности всех возможных ее значений [7]. 
И

$$
\psi_{T}(\tau)=b_{T 0}+b_{T 1} \tau+b_{T 2} \tau^{2}+b_{T 3} \tau^{3}+b_{T 4} \tau^{4}+b_{T 5} \tau^{5}+b_{T 6} \tau^{6},
$$

учитывая, что имеется программа машинного вычисления их коэффициентов через полиномы Чебышева [8].

Тогда

$$
\begin{gathered}
\partial \psi_{D}(\tau) / \partial \tau=b_{D 1}+2 b_{D 2} \tau+3 b_{D 3} \tau^{2}+4 b_{D 4} \tau^{3}+5 b_{D 5} \tau^{4}+6 b_{D 6} \tau^{5}, \\
\partial \psi_{T}(\tau) / \partial \tau=b_{T 1}+2 b_{T 2} \tau+3 b_{T 3} \tau^{2}+4 b_{T 4} \tau^{3}+5 b_{T 5} \tau^{4}+6 b_{T 6} \tau^{5}, \\
\partial^{2} \psi_{D}(\tau) / \partial \tau^{2}=2 b_{D 2}+6 b_{D 3} \tau+12 b_{D 4} \tau^{2}+20 b_{D 5} \tau^{3}+30 b_{D 6} \tau^{4} \\
\partial^{2} \psi_{T}(\tau) / \partial \tau^{2}=2 b_{T 2}+6 b_{T 3} \tau+12 b_{T 4} \tau^{2}+20 b_{T 5} \tau^{3}+30 b_{T 6} \tau^{4}
\end{gathered}
$$

Подставляя выражения (42), (43), (44), (45), (46) и (47) в решение (39), будем иметь:

$$
\begin{aligned}
& \psi(\xi, \tau)=\left[b_{D 0}+\left(b_{T 0}-b_{D 0}\right) \xi / \lambda\right]+\left[b_{D 1}+\left(b_{T 1}-b_{D 1}\right) \xi / \lambda\right] \tau+ \\
& \quad+\left(b_{D 2}+\left(b_{T 2}-b_{D 2}\right) \xi / \lambda\right] \tau^{2}+\left[b_{D 3}+\left(b_{T 3}-b_{D 3}\right) \xi / \lambda\right] \tau^{3}+ \\
& \quad+\left[b_{D 4}+\left(b_{T 4}-b_{D 4}\right) \xi / \lambda\right] \tau^{4}+\left[b_{D 5}+\left(b_{T 5}-b_{D 5}\right) \xi / \lambda\right] \tau^{5}+ \\
& \quad+\left[b_{D 6}+\left(b_{T 6}-b_{D 6}\right) \xi / \lambda\right] \tau^{6}+\sum_{i=1}^{\infty}\left[\left(2 \lambda^{2} \sin i \pi \xi / \lambda\right) /(i \pi)^{3}\right] \Pi_{i}(\tau),
\end{aligned}
$$

где

$$
\begin{gathered}
\Pi_{i}(\tau)=\left\{\left[( - 1 ) ^ { i } \left(b_{T 1}-2 b_{T 2}(\lambda / i \pi)^{2}+6 b_{T 3}(\lambda / i \pi)^{4}-24 b_{T 4}(\lambda / i \pi)^{6}+\right.\right.\right. \\
\left.+120 b_{T 5}(\lambda / i \pi)^{8}-720 b_{T 6}(\lambda / i \pi)^{10}\right)-\left(b_{D 1}-2 b_{D 2}(\lambda / i \pi)^{2}+\right. \\
\left.\left.+6 b_{D 3}(\lambda / i \pi)^{4}-24 b_{D 4}(\lambda / i \pi)^{6}+120 b_{D 5}(\lambda / i \pi)^{8}-720 b_{D 6}(\lambda / i \pi)^{10}\right)\right] \times \\
\times\left[1-\exp \left[-(i \pi / \lambda)^{2} \tau\right]\right]+2\left[( - 1 ) ^ { i } \left(b_{T 2}-3 b_{T 3}(\lambda / i \pi)^{2}+\right.\right. \\
\left.+4 b_{T 4}(\lambda / i \pi)^{4}-30 b_{T 5}(\lambda / i \pi)^{6}+180 b_{T 6}(\lambda / i \pi)^{8}\right)- \\
\left.-\left(b_{D 2}-3 b_{D 3}(\lambda / i \pi)^{2}+i 4 b_{D 4}(\lambda / i \pi)^{4}-30 b_{D 5}(\lambda / i \pi)^{6}+180 b_{D 6}(\lambda / i \pi)^{8}\right)\right] \tau+ \\
+3\left[(-1)^{i}\left(b_{T 3}-4 b_{T 4}(\lambda / i \pi)^{2}+20 b_{T 5}(\lambda / i \pi)^{4}-120 b_{T 6}(\lambda / i \pi)^{6}\right)-\right. \\
\left.-\left(b_{D 3}-4 b_{D 4}(\lambda / i \pi)^{2}+20 b_{D 5}(\lambda / i \pi)^{4}-120 b_{D 6}(\lambda / i \pi)^{6}\right)\right] \tau^{2}+ \\
+4\left[(-1)^{i}\left(b_{T 4}-5 b_{T 5}(\lambda / i \pi)^{2}+30 b_{T 6}(\lambda / i \pi)^{4}\right)-\right. \\
\left.-\left(b_{D 4}-5 b_{D 5}(\lambda / i \pi)^{2}+30 b_{D 6}(\lambda / i \pi)^{4}\right)\right] \tau^{3}+5\left[(-1)^{i}\left(b_{T 5}-6 b_{T 5}(\lambda / i \pi)^{2}\right)-\right. \\
\left.\left.-\left(b_{D 5}-6 b_{D 5}(\lambda / i \pi)^{2}\right)\right] \tau^{4}+6\left[(-1)^{i} b_{T 6}-b_{D 6}\right] \tau^{5}\right\} .
\end{gathered}
$$

Таким же образом градиент напора в месте разгрузки (40) представляется в виде

$$
\begin{gathered}
\partial \psi(\xi, \tau) /\left.\partial \xi\right|_{\xi=\lambda}=\left[\left(b_{T 0}-b_{D 0}\right)+\left(b_{T 1}-b_{D 1}\right) \tau+\left(b_{T 2}-b_{D 2}\right) \tau^{2}+\right. \\
+\left(b_{T 3}-b_{D 3}\right) \tau^{3}+\left(b_{T 4}-b_{D 4}\right) \tau^{4}+\left(b_{T 5}-b_{D 5}\right) \tau^{5}+ \\
\left.+\left(b_{T 6}-b_{D 6}\right) \tau^{6}\right] \lambda^{-1}+\sum_{i=1}^{\infty}\left[(-1)^{\left.i 2 \lambda /(i \pi)^{2}\right] \Pi_{i}(\tau)} .\right.
\end{gathered}
$$

Подставляя функцию (49) в формулу (41) и интегрируя ее в промежутке времени от 0 до $\tau$, получим выражение для определения безразмерных естественных ресурсов подземных вод: 


$$
\begin{gathered}
\Omega=\Phi K\left\{\left[\left(b_{D 0}-b_{T 0}\right) \tau-\left({ }^{1} / 2\right)\left(b_{T 1}-b_{D 1}\right) \tau^{2}-\left({ }^{1} / 3\right)\left(b_{T 2}-b_{D 2}\right) \tau^{3}-\right.\right. \\
-\left({ }^{1 / 4}\right)\left(b_{T 3}-b_{D 3}\right) \tau^{4}-\left({ }^{1 / 5}\right)\left(b_{T 4}-b_{D 4}\right) \tau^{5}-\left({ }^{1} / 6\right)\left(b_{T 5}-b_{D 5}\right) \tau^{6}- \\
\left.\quad-(1 / 7)\left(b_{T 6}-b_{D 6}\right) \tau^{7}\right] \lambda^{-1}-\sum_{i=1}^{\infty}\left[2 \lambda /(i \pi)^{2}\right]\left\{\left[\left(b_{T 1}-2 b_{T 2}(\lambda / i \pi)^{2}+\right.\right.\right. \\
\left.+6 b_{T 3}(\lambda / i \pi)^{4}-24 b_{T 4}(\lambda / i \pi)^{6}+120 b_{T 5}(\lambda / i \pi)^{3}-720 b_{T 6}(\lambda / i \pi)^{10}\right)- \\
\quad-(-1)^{i}\left(b_{D 1}-2 b_{D 2}(\lambda / i \pi)^{2}+6 b_{D 3}(\lambda / i \pi)^{4}-24 b_{D 4}(\lambda / i \pi)^{6}+\right. \\
\left.\left.+120 b_{D 5}(\lambda / i \pi)^{8}-720 b_{D 6}(\lambda / i \pi)^{10}\right)\right]\left[-(\lambda / i \pi)^{2}\left(1-\exp \left[-(i \pi / \lambda)^{2} \tau\right]\right)\right]+ \\
+\left[\left(b_{T 2}-3 b_{T 3}(\lambda / i \pi)^{2}+4 b_{T 4}(\lambda / i \pi)^{4}-30 b_{T 5}(\lambda / i \pi)^{6}+180 b_{T 6}(\lambda / i \pi)^{8}\right)-\right. \\
\quad-(-1)^{i}\left(b_{D 2}-3 b_{D 3}(\lambda / i \pi)^{2}+4 b_{D 4}(\lambda / i \pi)^{4}-30 b_{D 5}(\lambda / i \pi)^{6}+\right. \\
\left.\left.+180 b_{D 6}(\lambda / i \pi)^{8}\right)\right] \tau^{2}+\left[\left(b_{T 3}-4 b_{T 4}(\lambda / i \pi)^{2}+20 b_{T 5}(\lambda / i \pi)^{4}-\right.\right. \\
\left.-120 b_{T 6}(\lambda / i \pi)^{6}\right)-(-1)^{i}\left(b_{D 3}-4 b_{D 4}(\lambda / i \pi)^{2}+20 b_{D 5}(\lambda / i \pi)^{4}-\right. \\
\left.\left.-120 b_{D 6}(\lambda / i \pi)^{6}\right)\right] \tau^{3}+\left[\left(b_{T 4}-5 b_{T 5}(\lambda / i \pi)^{2}+30 b_{T 6}(\lambda / i \pi)^{4}\right)-\right. \\
\left.\quad-(-1)^{i}\left(b_{D 4}-5 b_{D 5}(\lambda / i \pi)^{2}+30 b_{D 6}(\lambda / i \pi)^{4}\right)\right] \tau^{4}+ \\
+\left[\left(b_{T 5}-6 b_{T 5}(\lambda / i \pi)^{2}\right)-(-1)^{i}\left(b_{D 5}-6 b_{D 5}(\lambda / i \pi)^{2}\right)\right] \tau^{5}+ \\
\left.+\left[b_{T 6}-(-1)^{i} b_{D 6}\right] \tau^{6}\right\} .
\end{gathered}
$$

Иногда возникает необходимость как можно точнее исследовать динамику некоторого частного случая, где граничные условия представлены колебаниями относительно большой амплитуды и частоты. При

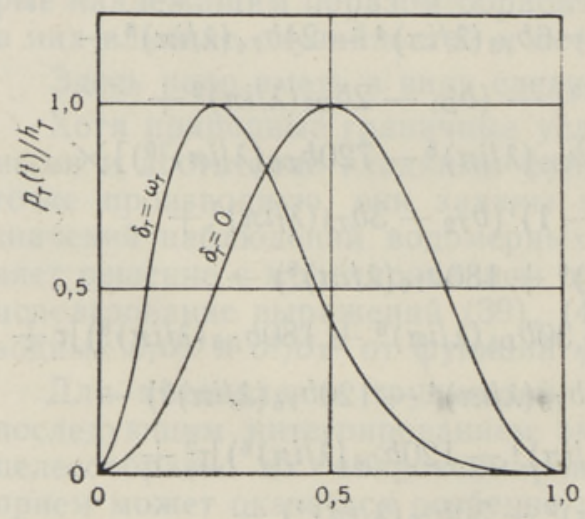

Рис. 2. Графики периодической функции (52) при $\delta_{T}=\omega_{T}$ и $\delta_{T}=0$. этом может появиться опасение, что аппроксимация полиномами даст неточные результаты. Тогда целесообразно прибегнуть к методам искусственного улучшения сходимости рядов Фурье.

В этом отношении особого внимания заслуживает метод $\sigma$-множителей [ $\left.{ }^{9}\right]$. Однако применение этого способа вычисления в наших целях требует дополнительных исследований, выходящих за пределы настоящей работы.

Для изучения многих теоретических вопросов режима подземных вод допустимо изображать природные граничные условия в виде периодических функций (рис. 2):

$$
p_{D}(t)=\left[N_{D} h_{D}\left(1-\cos \omega_{D} t\right) \exp \left[-\delta_{D} t\right]\right]+H
$$

и

$$
p_{T}(t)=N_{T} h_{T}\left(1-\cos \omega_{T} t\right) \exp \left[-\delta_{T} t\right],
$$

где $h_{D}$ и $h_{T}$ - амплитуды колебания напора соответственно в области питания и разгрузки; $H$ - превышение области питания над областью разгрузки; $\omega_{D}=2 \pi / \bar{t}_{D}, \omega_{T}=2 \pi / \bar{t}_{T} ; \bar{t}_{D}$ и $t_{T}$ - продолжительности периодов; $\delta_{D}, \delta_{T}, N_{D}$ и $N_{T}$ - коэффициенты, причем

$$
N_{D}=\left[\left(\delta_{D}^{2}+\omega_{D}^{2}\right) / 2 \omega_{D}^{2}\right] \exp \left[\left(2 \delta_{D} / \omega_{D}\right) \arctan \omega_{D} / \delta_{D}\right]
$$

H

$$
N_{T}=\left[\left(\delta_{T}^{2}+\omega_{T}^{2}\right) / 2 \omega_{T}^{2}\right] \exp \left[\left(2 \delta_{T} / \omega_{T}\right) \arctan \omega_{T} / \delta_{T}\right]
$$


При помощи надлежащим образом подобранных значений $H, h_{D}, h_{T}$, $N_{D}, N_{T}, \bar{t}_{D}, \bar{t}_{T}, \delta_{D}$ и $\delta_{T}$ можно варьировать граничные условия и после подстановки их в выражения (39), (40), (41) исследовать влияние их амплитуд, сдвига фаз и т. п. на фильтрационный режим потока.

Наконец обратим внимание на то, что функции $p_{D}(t)$ и $p_{T}(t)$ не обязательно связаны с областями питания и разгрузки потока подземных вод. Если условия изменения напора заданы при помощи буровых наблюдательных скважин (см. рис. 1, в), то закономерности режима фильтрации можно исследовать между любыми сечениями водоносного горизонта.

Автор выражает глубокую благодарность сотрудникам Института кибернетики АН ЭССР У. Нигулу и Т. Тобиасу за ценные указания.

\section{Л ИТЕРАТУ РА}

1. М аскет М., Течение однородных жидкостей в пористой среде, М.-Л., 1949.

2. Todd D. K., Ground Water Hydrology, New York, 1966.

3. К а р сл оу Г., Е гер Д., Теплопроводность твердых тел, М., 1964.

4. Щ елка чев В. Н., Л а пук Б. Б., Подземная гидравлика, М.-Л., 1949.

5. В а ллн ер Л. К., Оседание земной поверхности вследствие подземного водозабора на территории города Таллина, Современные движения земной коры, № 2, Тарту, 1965.

6. Куделин Б. И., Принципы региональной оценки естественных ресурсов подземных вод, Изд. Моск. ун-та, 1960.

7. Сборник определений основных гидрологических терминов и понятий, Под ред. А. И. Чеботарева, Л., 1957 (Главн. упр. гидрометеорологич. службы при СM СССР).

8. Пукк К., Приближение функции, заданной таблицей с постоянным шагом по методу наименьших квадратов при помощи ортогональных полиномов Чебышева, Программы для ЭЦВМ «Минск-2», Вып. 1, Таллин, 1965.

9. Л а нцош К., Практические методы прикладного анализа, М., 1961.
Институт геологии
Академии наук Эстонской ССР
Поступила в редакцию $10 / \mathrm{X} 1966$

\section{VALLNER}

\section{HARMOONILISE ANALUUSI KASUTAMINE UHEMÕOTMELISE SURVEPINNALISE PÖHJAVEE-VOOLU RÖU JA ÅRAVOOLU DUNAAMIKA UURIMISEKS}

Mittestatsionaarsele filtratsioonirežiimile alluv ühemõōtmeline survepinnaline põhjavee-vool on kirjeldatav võrrandiga (2) ja rajatingimustega (3) ning (4). Harmoonilise analüüsi abil on leitud avaldised pôhjavee-voolu hüdraulilise rōhu jaotuse (39), rōhu gradiendi (40) ja looduslike ressursside (41) väljendamiseks.

Mitteformaalse lahenduse eelduseks üldjuhul on rajatingimuste (3) kahekordne pidev diferentseeritavus aja järgi. Seetōttu soovitatakse empiiriliste funktsioonidena antud rajatingimusi lähendada Tšebōševi polünoomidega; kuuenda astme polünoomi puhul omandavad lahendid kuju (48), (49) ja (50). Teoreetiliste uurimiste jaoks vōib looduslikke rajatingimusi lähendada perioodiliste funktsioonidega (51) ning (52).

Antud töös kasutatud parameetrit $x$ on kirjanduses esitatud ka $x=T / S$ kujul, kus $S$ on mahtuvuse ja $T$ veejuhtivuse moodul. 
L. VALLNER

\section{APPLICATION OF HARMONIC ANALYSIS IN THE STUDY OF THE DYNAMICS OF THE PRESSURE AND DISCHARGE OF THE NONSTEADY ONE-DIMENSIONAL FLOW OF CONFINED GROUND WATER}

The nonsteady one-dimensional flow of confined ground water can be described by formula (2) as well as boundary conditions (3) and (4). With the application of methods of harmonic analysis, expressions have been found for the distribution of hydraulic pressure (39), gradient of pressure (40) and natural resources (41) of the flow of confined ground water.

The prerequisite for a nonformal solution in a general case is the continuous differentiability twice the boundary conditions (3). Therefore it is recommended to approximate the boundary conditions, given as empiric functions, with Chebyshev polynomials; in the case of the polynomial of the sixth power, the solutions obtain the forms (48), (49), and (50). For theoretical purposes, the natural boundary conditions can be approximated by periodical functions (51) and (52).

The parameter $x$ used in this paper has been presented in relevant literature also in the shape $x=T / S ; S$ being the coefficient of storage of aquifer, and $T-$ the coefficient of the transmissibility of the aquifer. 